\title{
HDAC6, chef d'orchestre de la défense anti-agrégats protéiques
}

\author{
Cyril Boyault, Saadi Khochbin
}

> HDAC6, une désacétylase particulière L'état d'acétylation des histones est contrôlé par une action antagoniste de deux familles d'enzymes: les histoneacétyltransférases (HAT), qui transfèrent un groupe acétyl de l'acétyl co-enzyme $A(\operatorname{co} A)$ sur le groupe $\varepsilon$-amine d'un résidu lysine spécifique; et les histonedésacétylases (HDAC) qui effectuent la réaction inverse. Les 18 HDAC identifiées à ce jour chez l'homme sont groupées dans différentes classes, qui tiennent compte également de leurs spécificités structurales et/ou catalytiques. Dans ce contexte, HDAC6 se distingue nettement des autres HDAC. En effet, les premières caractérisations fonctionnelles qui ont suivi sa découverte dans notre laboratoire [1], ont rapidement montré que HDAC6 possède des traits uniques, dont une localisation cytoplasmique et la capacité d'interagir avec l'ubiquitine. De plus, bien que la dénomination « histone désacétylase » soit utilisée, le premier substrat physiologique identifié d'HDAC6 fut l' $\alpha$-tubuline et non les histones. Ces caractéristiques ont entraîné la recherche de ses fonctions loin de la chromatine et de la transcription, cibles habituelles de l'action des HDAC [2]. Quant aux fonctions cellulaires impliquant HDAC6, les premières indications sont venues de l'identification de deux de ses partenaires cellulaires chez la souris (qui sont les protéines p97/VCP/ Cdc48 et UFD3/PLAP) et la démonstration de son interaction avec l'ubiquitine [3]. Ces données suggéraient l'implication d'HDAC6 dans la dégradation par le protéasome des protéines mal repliées dans la mesure où, chez la levure, les orthologues de ces partenaires d'HDAC6 (Cdc48 et UFD3) ont des fonctions bien établies dans la gestion des protéines mal repliées [4].

\section{HDAC6 et formation de l'agrésome}

Ces informations, ainsi que la découverte de l'association d'HDAC6 avec le complexe moteur dynéine/dynactine [5], ont ouvert la voie à la découverte de I'une des activités essentielles d'HDAC6 dans la défense cellulaire contre l'accumulation des agrégats de protéines mal repliées et hautement ubiquitinylées. Ce mécanisme de défense utilise un transport actif dépendant des microtubules pour acheminer les agrégats protéiques éparpillés dans la cellule vers une structure unique, connue sous le nom d'«agrésome » [6]. La formation d'un agrésome est le moyen pour la cellule de réduire la toxicité des microagrégats protéiques épars en facilitant leur prise en charge par d'autres mécanismes de défense cellulaire (voir plus loin) [6]. En 2003, le groupe du Dr Yao (Duke University, Durham NC, États-Unis) a montré qu'en interagissant simultanément avec les chaînes polyubiquitine des agrégats et avec le complexe moteur dynéine/ dynactine, HDAC6 assure la formation d'un agrésome [7].

Avant même la formation de l'agrésome, les agrégats bloquent l'activité du protéasome [8] et créent ainsi une toxicité qui persiste malgré la formation de l'agrésome. Fait intéressant, il a été montré très récemment qu'un dysfonctionnement du protéasome active un deuxième mécanisme de défense cellulaire qui consiste en la dégradation des
Inserm U823, Équipe épigénétique

et signalisation cellulaire,

Institut Albert Bonniot,

Rond-point de la Chantourne,

38706 La Tronche Cedex Grenoble, France.

Université Joseph Fourier, Institut Albert

Bonniot, 38700 Grenoble, France.

danielle.salas@ujf-grenoble.fr

agrégats protéiques par l'autophagie [9] $(\rightarrow)$ et HDAC6 a été identifiée comme un élément central dans l'interconnexion entre le dysfonc- $(\rightarrow)$ voir la Nouvelle de tionnement du J.Puyal et al., page 19 protéasome et de ce numéro

l'activation de l'autophagie [10].

\section{HDAC6, senseur de danger} et inducteur de la réponse de type « choc thermique » Ces travaux suggèrent fortement l'existence de mécanismes cellulaires nouveaux, impliquant HDAC6 et capables de «sentir» l'état de fonctionnement du protéasome. Notre équipe vient de décrire l'un de ces mécanismes, démontrant le rôle d'HDAC6 dans une troisième voie de défense cellulaire contre l'accumulation d'agrégats protéiques, aboutissant à l'induction d'une réponse de type « choc thermique » [11].

Les protéines dites «de choc thermique » ou HSP (heat shock protein) sont des chaperons moléculaires qui assurent le repliement correct des protéines et sauvegardent le protéome contre le danger de mauvais repliement et d'agrégation [12]. En présence d'un stimulus aboutissant à l'accumulation de protéines mal repliées, tels que choc thermique, hypoxie/ischémie, radicaux libres, déplétion en ATP, acidose et/ou dysfonction du protéasome, la fonction des HSP devient critique pour la survie de la cellule. Les HSP participent activement au désassemblage des agrégats ou s'opposent à leur formation et ainsi permettent la structuration correcte des protéines ou, à défaut, leur dégradation par le protéasome [13, 14]. 
Le facteur de transcription HSFl (heat shock factor 1) joue un rôle majeur dans l'activation des gènes codant pour les HSP. Dans les cellules non stressées, ce facteur se trouve sous une forme inactive dans un complexe avec le chaperon HSP90. À la suite d'un stress cytotoxique, ce complexe se dissocie et HSFl suit un processus d'activation lui permettant d'induire la transcription

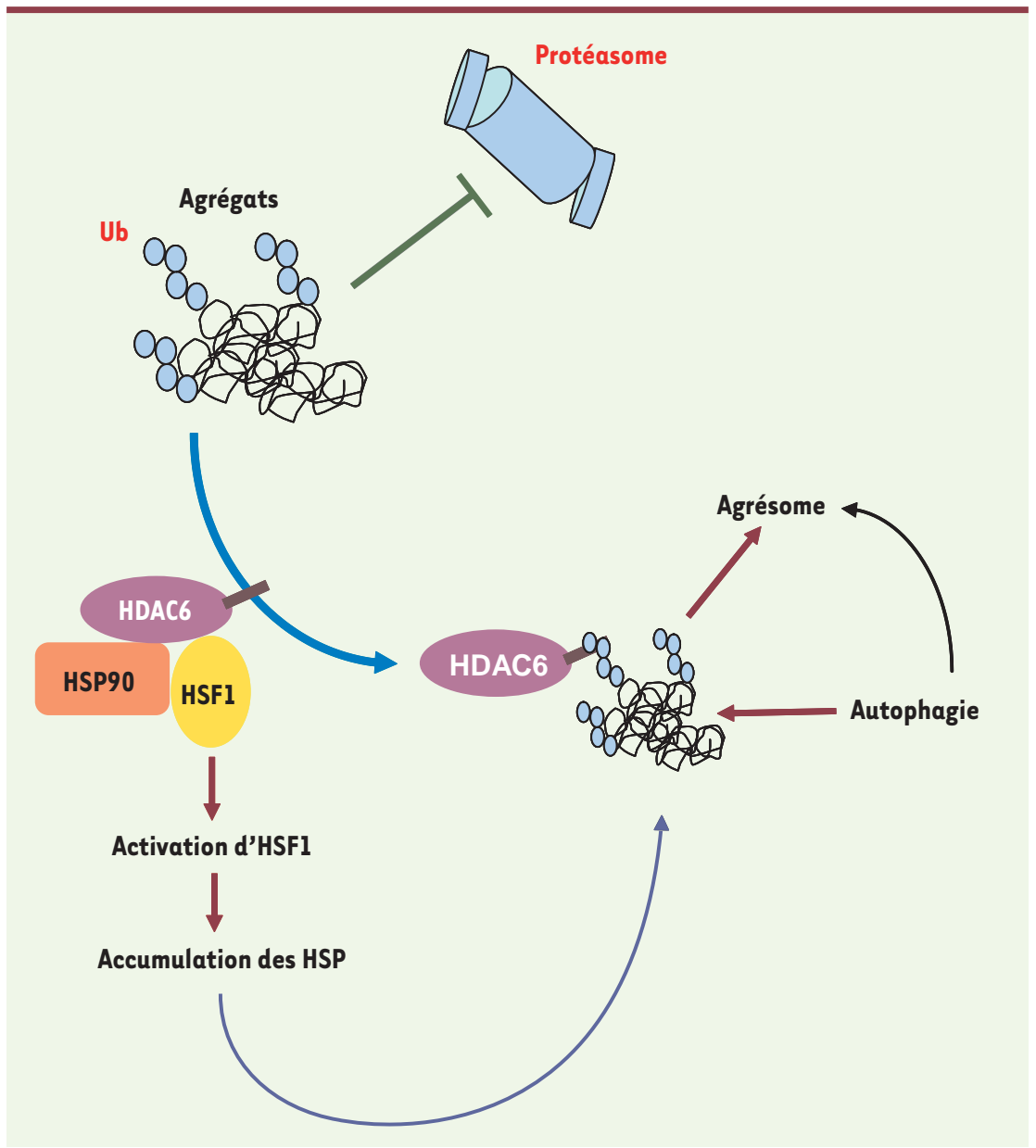

Figure 1. HDAC6 est un élément central dans la réponse cytoprotectrice des cellules contre l'accumulation d'agrégats protéiques. L'accumulation d'agrégats protéiques, par exemple dans le cas des maladies neurodégénératives, inhibe l'activité du protéasome amplifiant ainsi l'accumulation des protéines mal repliées et hautement ubiquitinylées dans la cellule. Les chaînes de polyubiquitine présentes au sein des agrégats «attrapent » HDAC6 via son domaine d'interaction avec I'ubiquitine. Cette interaction induit la dissociation d'HDAC6 du complexe HSP90, qui relargue à son tour le facteur de transcription HSFl. Ce facteur active la transcription de gènes codant pour les HSP : HSP70, HSP27, etc. HDAC6 ainsi libérée participe au transport des agrégats et assure la formation des agrésomes dans les cellules. HDAC6 induit également la dégradation des agrégats et de l'agrésome par autophagie. HDAC6 apparaît donc comme un chef d'orchestre de la réponse cytoprotectrice cellulaire contre l'accumulation des agrégats protéiques en induisant et coordonnant l'ensemble des voies permettant à la cellule de faire face à leur toxicité.
Or nous démontrons qu'HDAC6, également présente au sein du complexe HSP90-HSFl, a la capacité de «sentir » l'accumulation des protéines ubiquitinylées et, après interaction avec l'ubiquitine, peut induire la dissociation du complexe, ce qui libère HSFl, qui, activée, peut alors diriger la transcription des gènes HSP [11].

HDAC6 orchestre les trois mécanismes de protection en réponse à l'accumulation d'agrégats protéiques En résumé, l'ensemble de ces travaux montre qu'HDAC6 coordonne toutes les voies de réponse cellulaire à l'accumulation cytotoxique des agrégats protéiques. HDAC6 apparaît ainsi comme un surveillant attentif de l'activité du protéasome. Un dysfonctionnement de celui-ci conduit à l'accumulation de protéines ubiquitinylées, laquelle est «repérée » par HDAC6 qui interagit avec la chaîne de poly-ubiquitine et se dissocie du complexe HSP90. HDAC6 associée aux agrégats ubiquitinylés, interagit avec les moteurs moléculaires pour assurer leur transport le long des microtubules et la formation d'un agrésome. En même temps, elle active la dégradation de ces agrégats par autophagie, via un mécanisme qui reste à élucider (Figure 1). HDAC6 apparaît donc comme un véritable chef d'orchestre capable de «sentir» l'accumulation des protéines ubiquitinylées, d'induire et coordonner trois types de réponses cytoprotectrices.

\section{Conclusion}

Au vu de ces découvertes, il apparaît évident qu'HDAC6 pourrait se présenter comme une cible de choix dans la lutte contre les maladies neurodégénératives impliquant l'accumulation pathologique d'agrégats protéiques. En effet, l'utilisation de systèmes modèles avec les protéines mutées impliquées dans ces maladies, telles que l'Huntingtine ou le récepteur aux androgènes, indique très clairement un rôle déterminant pour HDAC6 dans l'élimination des agrégats 
et la réduction de leur toxicité [16]. Le renforcement des différentes activités d'HDAC6 dans la formation des agrésomes, dans la dégradation autophagique des agrégats et dans l'activation des gènes HSP, pourrait offrir des solutions thérapeutiques prometteuses dans la lutte contre ces maladies, mais avant cela, il est important de mieux comprendre les mécanismes sous-jacents à l'action d'HDAC6 dans ces processus. $\varepsilon n$ effet, les activités cytoprotectrices d'HDAC6 en réponse à l'accumulation des agrégats protéiques font appel à sa capacité à reconnaître l'ubiquitine et à son activité catalytique. Alors que le rôle du domaine de reconnaissance de l'ubiquitine d'HDAC6 dans la détection des agrégats protéiques et l'activation d'HSFl est relativement bien compris, le rôle de son activité catalytique dans la formation d'agrésomes et dans l'autophagie reste complètement obscur.

Il est par conséquent nécessaire de rester prudent sur les stratégies à adopter. En effet, il a récemment été montré, contre toute attente, que l'inhibition de l'activité tubuline-désacétylase d'HDAC6 restaure le trafic intracellulaire dépendant des moteurs moléculaires, dynéine et kinésine1 , perturbé dans les neurones affectés par la maladie d'Hungtinton $[17,18]$.

Un ciblage spécifique de différentes activités d'HDAC6 devrait donc être envisagé pour obtenir des effets optimaux dans les approches thérapeutiques des maladies neurodégénératives visant cette protéine. $\diamond$

HDAC6 orchestrates

cell defense responses against

cytotoxic protein aggregates

\section{RéFÉRENCES}

1. Verdel A, Khochbin S. Identification of a new family of higher eukaryotic histone deacetylases. Coordinate expression of differentiation-dependent chromatin modifiers. J Biol Chem 1999; $274: 2440-5$.

2. Boyault C, Sadoul K, Pabion M, Khochbin S. HDAC6, at the crossroads between cytoskeleton and cell signaling by acetylation and ubiquitination. Oncogene 2007; $26: 5468-76$.

3. Seigneurin-Berny D, Verdel A, Curtet $S$, et al. Identification of components of the murine histone deacetylase 6 complex: link between acetylation and ubiquitination signaling pathways. Mol Cell Biol 2001 ; $21: 8035-44$.

4. Rumpf $S$, Jentsch S. Functional division of substrate processing cofactors of the ubiquitin-selective $\mathrm{Cdc} 48$ chaperone. Mol Cell 2006; $21: 261-9$.

5. Hubbert C, Guardiola A, Shao R, et al. HDAC6 is a microtubule-associated deacetylase. Nature 2002 ; 417 : 455-8.

6. Kopito RR. Aggresomes, inclusion bodies and protein aggregation. Trends Cell Biol $2000 ; 10$ : 524-30.
7. Kawaguchi Y, Kovacs JJ, McLaurin A, et al. The deacetylase HDAC6 regulates aggresome formation and cell viability in response to misfolded protein stress. Cell 2003; $115: 727-38$.

8. Bennett $\varepsilon$ J, Bence NF, Jayakumar R, Kopito RR. Global impairment of the ubiquitin-proteasome system by nuclear or cytoplasmic protein aggregates precedes inclusion body formation. Mol Cell 2005; 17 : 351-65.

9. Puyal J, Ginet V, Vaslin A, Clarke PGH.

L'autophagie remplaçant de luxe du protéasome. Med Sci (Paris) 2008; $24: 19-21$.

10. Pandey UB, Nie Z, Batlevi Y, et al. HDAC6 rescues neurodegeneration and provides an essential link between autophagy and the UPS. Nature 2007 ; $447: 859-63$.

11. Boyault C, Zhang Y, Fritah S, et al. HDAC6 controls major cell response pathways to cytotoxic accumulation of protein aggregates. Genes Dev 2007 $21: 2172-81$.

12. Arrigo AP. Chaperons moléculaires et repliement des protéines: l'exemple de certaines protéines de choc ther. Med Sci (Paris) $2005 ; 21: 619-25$.

13. Whitesell L, Lindquist SL. HSP90 and the chaperoning of cancer. Nat Rev Cancer $2005 ; 5: 761-72$.

14. Andermarcher $\varepsilon$, Bossis G, Farras R, et al. La dégradation protéasomique: de l'adressage des protéines aux nouvelles perspectives thérapeutiques. Med Sci (Paris) 2005 ; 2 : 141-9.

15. Voellmy R. On mechanisms that control heat shock transcription factor activity in metazoan cells. Cell Stress Chaperones 2004 ; 9 : 122-33.

16. Rubinsztein DC. Autophagy induction rescues toxicity mediated by proteasome inhibition. Neuron 2007; $54: 854-6$.

17. Dompierre JP, Godin JD, Charrin BC, et al. Histone deacetylase 6 inhibition compensates for the transport deficit in Huntington's disease by increasing tubulin acetylation. J Neurosci 2007 ; 27 : 3571-83.

18. Lievens JC, Birman S. La chorée de Huntington chez la drosophile et chez la souris: vers de nouvelles pistes thérapeutiques? Med Sci (Paris) 2003; 19 : 593-9.

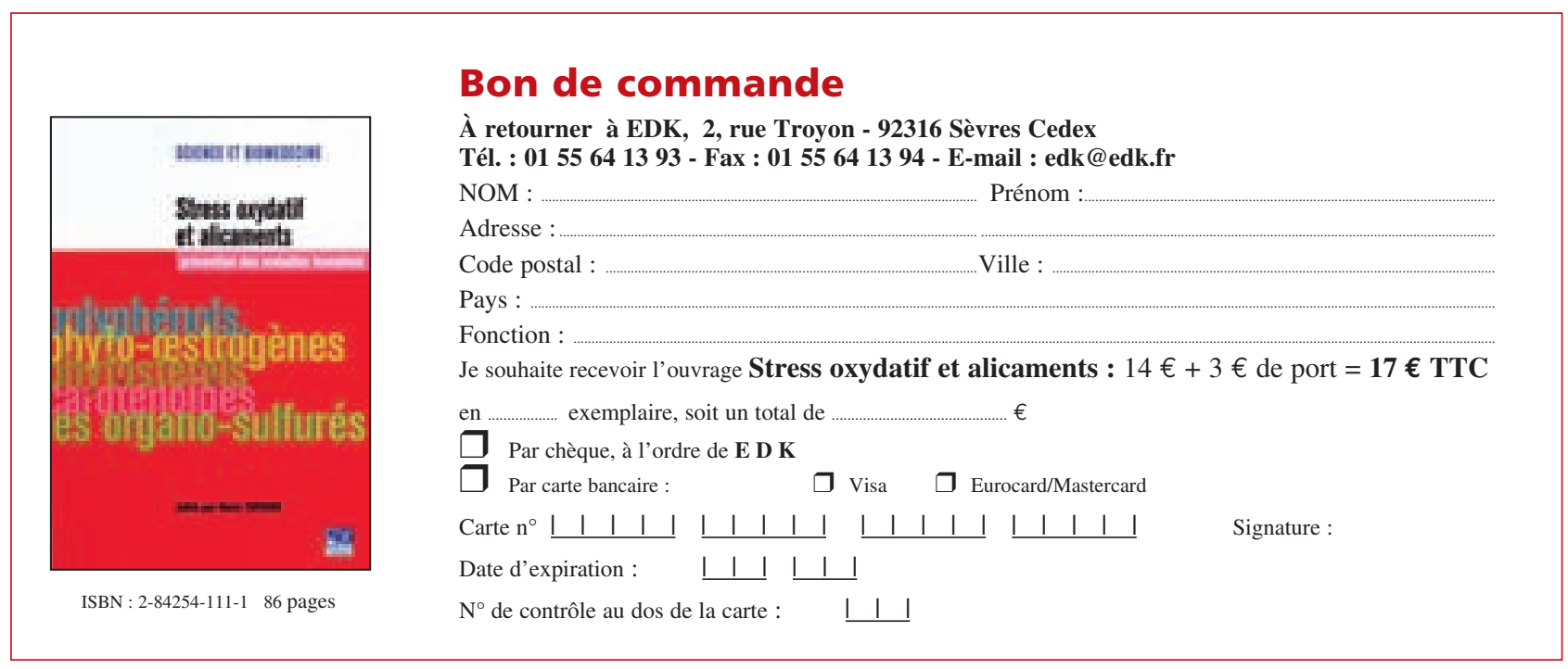

